\title{
Ultrahigh-Energy multi-messengers at the Pierre Auger Observatory
}

\author{
Francisco Pedreira $^{1, a}$ for the Pierre Auger Collaboration ${ }^{2, b}$ \\ ${ }^{1}$ University of Santiago de Compostela, 15705,Santiago de Compostela, Spain \\ ${ }^{2}$ Avda. San Martín Norte, Malargüe, Argentina
}

\begin{abstract}
The study of correlations between observations of different messengers from extreme sources of the Universe has emerged as an outstanding way to make progress in astrophysics. The Pierre Auger Observatory is capable of significant contributions as an ultra-high energy particle detector, particularly through its capability to search for inclined showers produced by neutrinos. We describe the neutrino searches made with the Observatory with particular emphasis on the recent results following the detections of gravitational waves from binary mergers with Advanced LIGO and VIRGO, leading to competitive limits.
\end{abstract}

\section{Introduction}

The discovery of gravitational waves (GW) events by LIGO and in particular from the binary neutronstar (NS) merger GW170817 is one of the most important milestones in multi-messenger astronomy. The Pierre Auger Observatory plays a relevant role in the field of multi-messenger astronomy because with Auger we can detect Ultra-High Energy (UHE) neutrons, photons and neutrinos. In this contribution, we are going to focus on the latter, the neutrinos.

\section{Search for neutrinos with Auger}

\subsection{Neutrino searching basics}

The way to identify neutrinos is explained in the next lines. Protons, heavier nuclei, and photons interact shortly after entering the atmosphere, while neutrinos can initiate showers deep in the atmosphere. At large zenith angles, the atmosphere is thick enough so that the electromagnetic component of nucleonic cosmic rays gets absorbed, and the shower front at ground level is mainly composed of muons ("old" shower front). On the other hand, showers induced by neutrinos deep in the atmosphere have a considerable electromagnetic component at the ground ("young" shower front).

With the Pierre Auger Observatory we can detect neutrinos exploiting two different channels [1]. In the "downward-going" (DG) channel, showers with a large zenith angle (higher than $60^{\circ}$ ) are selected. In the "Earth-skimming" (ES) channel, we search for tau neutrinos that travel in a slightly

\footnotetext{
a e-mail: francisco.pedreira@rai.usc.es

${ }^{b}$ Full author list and acknowledgments at: http://auger.org/archive/authors_2018_09.html
} 
upward direction with respect to the ground, that can interact close to the surface producing a tau lepton which escapes the Earth and decays in the atmosphere, close to the Auger surface detector (SD) of water-cherenkov detector (WCD).

The way to look for inclined showers is to use the shape of the pattern of triggered WCD on ground. These showers induce an elliptical elongated footprint on the ground where the apparent propagation speed of the signal is close to the speed of light. The second step is to look for "young" showers, meaning to look for signals with electromagnetic component. In the $\sim 1600$ WCD stations of the SD of the Pierre Auger Observatory, the signals produced by the shower particles are digitized with Flash Analog to Digital Converters with 25 ns resolution. This digitization allows us to distinguish signals induced by inclined showers initiated high in the atmosphere from the signals expected in inclined showers initiated close to the ground. The first of them appears as peaked signals narrow in time, but "young" showers induce signals more extended in time. We can distinguish them using the average value of the AreaOverPeak $(\langle A o P\rangle)$. The AoP is the total signal recorded by each WCD divided by the peak value. In an "old" shower the $\langle A o P\rangle$ is close to 1 , but in a "young" shower values of $\langle A o P\rangle>1$ are expected according to MC simulations of UHE $v$-induced showers.

\subsection{Data unblinding and limits}

No candidates for neutrino-induced showers were found after imposing all the selection criteria (see Ref. [2] for full details) to the data collected between 1 January 2004 and 31 March 2017 in the Earth-skimming and downward-going channels.

Assuming a differential neutrino flux $d N\left(E_{v}\right) / d E_{v}=k \cdot E_{v}^{-2}$ and a $v_{e}: v_{\mu}: v_{\tau}=1: 1: 1$ flavor ratio, an upper limit on the value of $k$ can be obtained as:

$$
k=\frac{N_{u p}}{\int_{E_{v}} E_{v}^{-2} \varepsilon_{t o t}\left(E_{v}\right) d E_{v}},
$$

where $N_{u p}$, the actual value of the upper limit on the signal events depends on the number of observed events and expected background, as well as on the confidence level required (90\% C.L. in our case), and $\varepsilon_{t o t}$ is the total exposure as a function of the neutrino energy.

The result is plotted in Figure 1 (left), and the numerical value of the limit that we obtained is:

$$
k \sim 5.0 \times 10^{-9} \mathrm{GeV} \mathrm{cm}^{-2} \mathrm{~s}^{-1} \mathrm{sr}^{-1} .
$$

As the Pierre Auger Observatory has a good angular resolution (less than 2.5 $)$ [3], we can also obtain a limit to point-like sources of UHE $v$. Following a similar procedure, we calculated the upper limit to point-like sources, and the result is shown in Figure 1 (right). It is important to point out that with Auger SD we are most sensitive to neutrinos in the ES channel. For that reason, we are particularly sensitive to sources at declinations that are wiewed by Auger with zenith angles between $90^{\circ}$ and $95^{\circ}$.

\section{Gravitational wave events}

\subsection{First gravitational wave}

In 2015 LIGO and Virgo detected two gravitational wave events caused by the merger of two blackholes [4]. The Pierre Auger Observatory searched for neutrinos in coincidence with these GW events. We applied the Earth-skimming and downward-going neutrino selection criteria to data collected $\pm 500 \mathrm{~s}$ around GW events and one day after GW events [5]. No candidates for neutrino showers were found, and we placed constraints on the amount of energy radiated in the form of UHEv. 


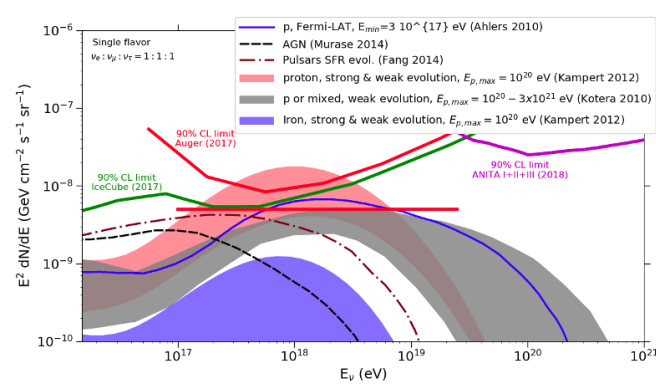

(a) Limit to the diffuse flux

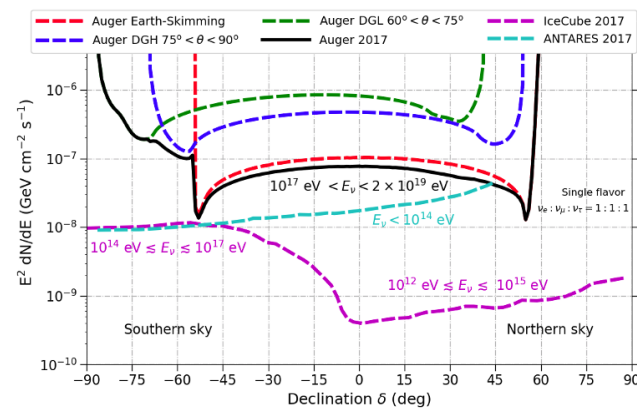

(b) Limit to point-like sources

Figure 1: Auger SD upper limits to fluxes of UHEv for the period 01 January 2004 to 31 March 2017 [2].

\subsection{Follow-up of GW170817}

The most important event in multi-messenger astronomy was the neutron star-neutron star merger GW170817. It was observed not only in gravitational waves, but also in radio, optical and X-rays. Moreover, Fermi detected a gamma-ray burst in coincidence with the GW detected by LIGO and Virgo [6].

ANTARES, IceCube and the Pierre Auger Collaboration searched for neutrinos in coincidence with GW170817 [7]. In Figure 2 we plot the skymap of the GW event at the moment of emission. The source of GW170817 was in an optimal position in the sky for the Pierre Auger Observatory, in the field of view of the ES channel, which is the most sensitive one in Auger.

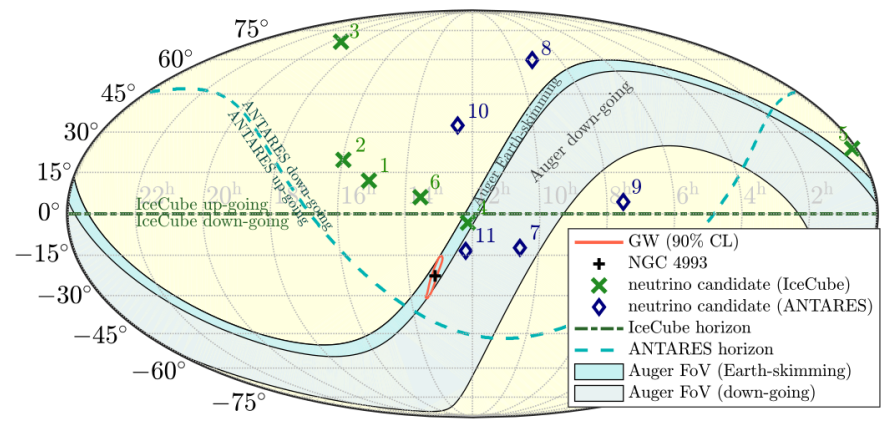

Figure 2: Localizations and sensitive sky areas for ANTARES, IceCube and Auger at the time of the GW170817 event in equatorial coordinates [7].

No candidates were found by any of the observatories. In Figure 3 we show the neutrino fluence limits for $\pm 500 \mathrm{~s}$ and 14 days time-window where the searches were performed. The limit that the Pierre Auger Collaboration achieves is complementary to the Icecube and Antares limits. Despite the fact that Auger is not a dedicated $v$ experiment, produced a remarkably good limit at EeV energies. However, with Auger we cannot see the source all the time in the inclined directions where we search 
for UHE $v$ due to the rotation of the Earth, and this is the reason why the limit in the 14 days timewindow is one order of magnitude higher than the IceCube one.

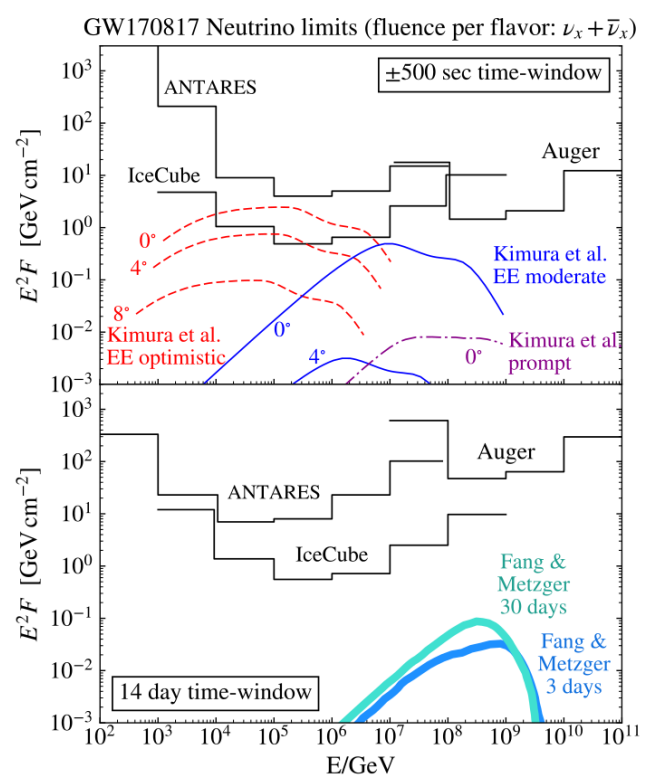

Figure 3: Upper limits (at $90 \%$ C.L.) on the neutrino spectral fluence from GW170817 during a $\pm 500 \mathrm{~s}$ window centered on the GW trigger time (top panel), and a 14-day window following the GW trigger (bottom pannel) [7].

\section{Conclusions}

Multi-messenger astronomy promises to give extraordinary insights into the working of the most extreme Universe, and all collaborations detecting particles and radiation should work together.

The Pierre Auger Observatory is a key detector in the multi-messenger astronomy at EeV energies due to the different kind of particles it can detect (photons, neutrons, and especially neutrinos). Indeed, with the Auger SD we have a very good sensitivity to UHE neutrinos, a large fraction of the sky can be probed with a good angular resolution.

\section{References}

[1] A. Aab et al. (Pierre Auger Collaboration), Phys. Rev. D 91, 092008 (2015)

[2] E. Zas for the Pierre Auger Collaboration, PoS (ICRC 2017), 972 (2017)

[3] A. Aab et al. (Pierre Auger Collaboration), JCAP 08, 019 (2014)

[4] B. P. Abbott et al. (LIGO Scientific and Virgo Collaborations), Phys. Rev. Lett. 116, 061102 (2016)

[5] A. Aab et al. (Pierre Auger Collaboration), Phys. Rev. D. 94, 122007 (2016)

[6] LIGO Scientific Collaboration et al., ApJL 848, L12 (2017)

[7] A. Albert et al. (ANTARES, IceCube, Pierre Auger, LIGO Scientific and Virgo Collaborations), ApJL 850, L35 (2017) 